\title{
MÉDIA DIÁRIA DO DÉFICIT DE PRESSÃO DE SATURAÇÃO DO VAPOR D’ÁGUA DO AR E SUA INFLUÊNCIA NA EVAPOTRANSPIRAÇÃO DE REFERÊNCIA PELO MODELO DE PENMAN-MONTEITH (FAO 56) EM PIRACICABA - SP
}

\author{
GUSTAVO B. LYRA ${ }^{1}$, ANTONIO R. PEREIRA ${ }^{2}$, GILBERTO C. SEDIYAMA ${ }^{3}$, \\ GUILHERME B. LYRA ${ }^{4}$, ALAILSON V. SANTIAGO ${ }^{1}$, MARCOS V. FOLEGATTI ${ }^{5}$
}

RESUMO: Utilizando-se como padrão as medidas lisimétricas de um gramado, avaliaram-se os resultados de estimativas de $\mathrm{ET}_{\mathrm{o}}$ obtidas com $\mathrm{D}_{\text {ar_méd }}$ oriundos de 14 métodos para seu cálculo. Tais comparações permitiram separar os métodos de cálculo de $\mathrm{D}_{\text {ar_méd }}$ em três grupos, ou seja: um grupo resultou sempre em superestimativa de $\mathrm{ET}_{0}$; em outro, a tendência foi de sempre subestimar, e no último a tendência foi de superestimar valores baixos $\left(<4,5 \mathrm{~mm} \mathrm{dia}^{-1}\right)$ e de subestimar valores altos de $\mathrm{ET}_{\mathrm{o}}$. Não foram observadas diferenças significativas (teste $\mathrm{t} ; \mathrm{p}<0,05$ ) de $\mathrm{ET}_{\mathrm{o}}$, em função dos métodos de $\mathrm{D}_{\mathrm{ar} \_ \text {méd }}$ ao utilizar a média horária da temperatura e/ou umidade relativa do ar em relação aos determinados com a média dos valores máximo e mínimo desses elementos. Dos métodos propostos pelo boletim FAO56, apenas o que utilizou a umidade relativa média para calcular a pressão parcial de vapor d'água $\left(e_{a}\right)$ apresentou estimativa satisfatória. As melhores estimativas de $\mathrm{ET}_{\mathrm{o}}$ foram obtidas com os métodos de $\mathrm{D}_{\text {ar_méd, }}$ que utilizaram média da temperatura do ar na determinação da pressão de saturação $\left(\mathrm{e}_{\mathrm{s}}\right)$, e média da umidade relativa para $e_{a}$. A utilização do déficit de pressão de saturação em um único horário $(9$ e 10 $\mathrm{h}$ local), como representativo para o $\mathrm{D}_{\mathrm{ar} \_ \text {méd, }}$ mostrou-se boa alternativa na estimativa da $\mathrm{ET}_{\mathrm{o}}$, para as condições climáticas de Piracicaba - SP.

PALAVRAS-CHAVE: pressão de saturação do vapor d'água, equação combinada, análise de sensibilidade.

\section{DAILY AVERAGE OF THE SATURATION-VAPOR-PRESSURE DEFICIT OF THE AIR AND ITS INFLUENCE UPON THE REFERENCE EVAPOTRANSPIRATION BY PENMAN- MONTEITH MODEL (FAO56) IN PIRACICABA, SP, BRAZIL}

SUMMARY: The results of the evapotranspiration obtained with the $\mathrm{D}_{\text {air_avg }}$ proceeding from 14 methods were evaluated, by using the lysimetric measures of a sward. These comparisons allowed to separate the $\mathrm{D}_{\text {ar_avg }}$ calculating methods into three groups: a group always resulted into an overestimate $\mathrm{ET}_{0}$; in other one always tended to underestimating the $\mathrm{ET}_{0}$; and in the last tended to overestimating the low values $\left(<4.5 \mathrm{~mm} \mathrm{day}^{-1}\right)$, while underestimating the high values of $\mathrm{ET}_{\mathrm{o}}$. No significant statistical differences were observed ( $\mathrm{t}$ test; $\mathrm{p}<0.05)$ in $\mathrm{ET}_{\mathrm{o}}$ as a function of the $\mathrm{D}_{\text {air_arg }}$ methods, when using the hourly average of the temperature and/or the air relative humidity in relation to those determined with the average of the maximum and minimum values of these elements. Among the methods proposed by paper FAO56, just the one using the average relative humidity to calculate the actual pressure $\left(e_{a}\right)$ showed a satisfactory estimate. The best estimates of $\mathrm{ET}_{\mathrm{o}}$ were obtained with the $\mathrm{D}_{\text {air_avg }}$ methods using the average of the air temperature in the determination of the saturation pressure $\left(e_{s}\right)$ and the average of the relative humidity for the actual pressure $\left(\mathrm{e}_{\mathrm{a}}\right)$. The use of saturation-vapor-pressure deficit of the air at a single time (from 9 or 10 a.m. local) as a representative of $\mathrm{D}_{\text {air_avg }}$ showed to be a satisfactory alternative in estimating the $\mathrm{ET}_{\mathrm{o}}$ under the climatic conditions of Piracicaba, SP, Brazil.

KEYWORDS: saturation vapor pressure, combination equation, sensitivity analysis

\footnotetext{
${ }^{1}$ Meteorologista, Doutorando em Física do Ambiente Agrícola, Departamento de Ciências Exatas, ESALQ/USP, Av. Pádua Dias, 11, Piracicaba - SP, e-mail: gblyra@esalq.usp.br

${ }^{2}$ Eng $^{\text {o }}$ Agrônomo, Prof. Titular, Depto. de Ciências Exatas, ESALQ/USP, Piracicaba - SP, e-mail: arpereir@esalq.usp.br

${ }^{3}$ Eng $^{\mathrm{o}}$ Agrônomo, Prof. Titular, Depto. de Engenharia Agrícola, UFV, Viçosa - MG, e-mail: sediyama@ufv.br

${ }^{4}$ Eng $^{\circ}$ Agrônomo, Doutorando em Produção Vegetal, LEA/UENF, Campos dos Goytacazes - RJ, e-mail: gbastos@uenf.br

${ }^{5}$ Eng ${ }^{\circ}$ Agrônomo, Prof. Associado, Depto. de Engenharia Rural, ESALQ/USP, Piracicaba - SP, e-mail: mvfolega@esalq.usp.br

Recebido pelo Conselho Editorial em: 12-6-2003

Aprovado pelo Conselho Editorial em: 21-5-2004
} 


\section{INTRODUÇÃO}

Um elemento meteorológico importante para a estimativa da evapotranspiração de referência $\left(\mathrm{ET}_{\mathrm{o}}\right)$ por Penman-Monteith é o déficit de pressão de saturação do vapor d'água do ar $\left(\mathrm{D}_{\mathrm{ar}}\right)$. O método utilizado na determinação da média diária de $\mathrm{D}_{\mathrm{ar}}\left(\mathrm{D}_{\text {ar_méd }}\right)$ pode introduzir erros significativos nos valores de $\mathrm{ET}_{\mathrm{o}}$ diária (DOORENBOS \& PRUITT, 1975). Isso se deve, principalmente, à nãolinearidade da relação entre pressão de saturação do vapor d'água $\left(e_{s}\right)$ e temperatura do ar.

Vários métodos de cálculo de $\mathrm{D}_{\text {ar_méd }}$ são descritos na literatura (JENSEN, 1974; DOORENBOS \& PRUITT, 1975; SADLER \& EVANS, 1989; JENSEN et al., 1990; HOWELL \& DUSEK, 1995; PEREIRA et al., 1997 a; ALLEN et al., 1998), sendo que alguns não são práticos, e suas estimativas diferem estatisticamente entre si (PEREIRA et al., 1997 b). Diferenças de -26 a $+3 \%$ entre os valores de $\mathrm{D}_{\text {ar_méd }}$ calculados com dados horários e estimados por métodos aproximados foram determinados por JENSEN (1974). Utilizando cinco métodos, DOORENBOS \& PRUITT (1975) relatam subestimativa de $32 \%$ em média. Aqueles propostos pelo manual ASCE nº 70 (JENSEN et al., 1990) apresentam erros na faixa de 4,4\% a 36,4\% (HOWELL \& DUSEK, 1995).

PEREIRA et al. (1997 b) avaliaram a possibilidade de utilização de apenas um valor horário de $\mathrm{D}_{\mathrm{ar}}$ (entre 9 e $10 \mathrm{~h}$ local), como representativo para $\mathrm{D}_{\mathrm{ar} \_ \text {méd, }}$ em Piracicaba - SP. Observaram que os valores de $D_{a r}$ das $10 \mathrm{~h}$ superestimaram o $\mathrm{D}_{\text {ar_méd }}$ em $11,7 \%$, enquanto os das $9 \mathrm{~h}$ subestimaram em $27,5 \%$, em média.

Estudando o efeito de 15 métodos de estimativa de $\mathrm{D}_{\text {ar_méd }}$ sobre a estimativa de $\mathrm{ET}_{\mathrm{o}}$, usando a equação de Penman modificada por Bavel (BAVEL, 1966), SADLER \& EVANS (1989) relatam variaçães de 8 a $17 \%$ em relação ao melhor método de $\mathrm{D}_{\text {ar_méd }}$ na determinação de $\mathrm{ET}_{\mathrm{o}}$. PEREIRA et al. (1996), baseados em PRUITT \& DOORENBOS (1977), citam erros relativos de 10 a $50 \%$ no inverno, e em torno de $10 \%$ no verão, com alguns casos extremos acima de $100 \%$, na estimativa de $\mathrm{ET}_{\mathrm{o}}$ pela equação original de Penman, ao utilizarem seis métodos de cálculo de $\mathrm{D}_{\text {ar_méd. }}$.

O objetivo do presente trabalho foi avaliar a influência de métodos para a determinação da média diária do déficit de pressão de saturação do ar, inclusive aqueles descritos em ALLEN et al. (1998), na estimativa da evapotranspiração de referência, padrão FAO56.

\section{MATERIAL E MÉTODOS}

O experimento foi conduzido no período de $1^{\circ}-01$ a 31-12-1996, numa área de $3.150 \mathrm{~m}^{2}(35 \mathrm{x}$ 90 m), localizada na Fazenda Areão, ESALQ/USP, em Piracicaba - SP (latitude 22 $42^{\prime}$ 'S; longitude $47^{\circ} 30^{\prime} \mathrm{W}$ e altitude de $546 \mathrm{~m}$ ). Pela classificação de Köeppen, o clima da região é do tipo Cwa (subtropical úmido, com chuvas no verão e inverno seco). O solo local foi classificado como Terra Roxa Estruturada (Alfisol), com declividade média de 2,3\%, e vegetado com grama batatais (Paspalum notatum Flügge) (SANTIAGO et al., 2002).

A evapotranspiração de referência diária foi determinada pela variação da massa de um lisímetro de pesagem gramado $\left(\mathrm{ET}_{\text {o_lis }}, \mathrm{mm} \mathrm{d}^{-1}\right.$ ), descrito em SILVA et al. (1999 b). Detalhes do seu manejo são apresentados por SILVA et al. (1999 a). Devido a problemas operacionais com o lisímetro de pesagem durante a estação chuvosa e em dias secos com rajadas de vento, como descrito por PEREIRA et al. (2002), foram selecionados apenas 141 dias para as análises.

A estimativa de $\mathrm{ET}_{\mathrm{o}}$ diária $\left(\mathrm{mm} \mathrm{d}^{-1}\right)$ seguiu as parametrizações do modelo de Penman-Monteith descritas em ALLEN et al. (1998), ou seja: 


$$
\mathrm{ET}_{\mathrm{o}}=\frac{0,408 \mathrm{~s}\left(\mathrm{R}_{\mathrm{n}}-\mathrm{G}\right)+\gamma \frac{900}{\mathrm{~T}_{\text {méd }}+273} \mathrm{u}_{2} \mathrm{D}_{\text {ar_méd }}}{\mathrm{s}+\gamma\left(1+0,34 \mathrm{u}_{2}\right)}
$$

em que,

$\mathrm{T}_{\text {méd }}$ - temperatura média do ar a $2 \mathrm{~m}$ de altura $\left[\mathrm{T}_{\text {méd }}=\left(\mathrm{T}_{\text {máx }}+\mathrm{T}_{\text {mín }}\right) / 2\right],{ }^{\circ} \mathrm{C}$;

$\mathrm{T}_{\text {máx }}$ e $\mathrm{T}_{\text {mín }}$ - temperatura máxima e mínima do ar, respectivamente, ${ }^{\circ} \mathrm{C}$;

$\mathrm{s}$ - derivada da curva de pressão de saturação do vapor do ar no ponto de $\mathrm{T}_{\text {méd }}$ [s $=4098(0,6108$ $\left.\left.\exp \left(17,27 \mathrm{~T}_{\text {méd }} / 237,3+\mathrm{T}_{\text {méd }}\right)\right) /\left(\mathrm{T}_{\text {méd }}+237,3\right)^{2}\right], \mathrm{kPa}^{\circ} \mathrm{C}^{-1}$;

$\mathrm{R}_{\mathrm{n}}$ - saldo de radiação total diário estimado (ALLEN et al., 1998), $\mathrm{MJ} \mathrm{m}^{-2} \mathrm{dia}^{-1}$;

$\mathrm{G}$ - fluxo de calor no solo $\left(\mathrm{G}=0\right.$ ), na escala diária (ALLEN et al., 1998), $\mathrm{MJ} \mathrm{m}^{-2} \mathrm{dia}^{-1}$;

$\gamma$ - coeficiente psicrométrico, $\mathrm{kPa}^{\circ} \mathrm{C}^{-1}$;

$\mathrm{u}_{2}$ - velocidade do vento a $2 \mathrm{~m}$ de altura, $\mathrm{m} \mathrm{s}^{-1}$;

$\mathrm{D}_{\text {ar_méd }}$ - média diária do déficit de pressão de saturação do vapor d'água do ar, kPa.

$\mathrm{O} \gamma$ foi admitido como constante e igual a $0,0633 \mathrm{kPa}{ }^{\circ} \mathrm{C}^{-1}$, devido à pequena amplitude da pressão atmosférica local ao longo do ano, com um máximo de $95,7 \mathrm{kPa}$ em julho e mínimo de $95,03 \mathrm{kPa}$ em janeiro. Assumiu-se o calor latente de evaporação da água igual a 2,45 $\mathrm{MJ} \mathrm{kg}^{-1}$, e os métodos de estimativa de $\mathrm{D}_{\text {ar_méd }}$ analisados foram divididos em seis grupos (Tabela 1).

Uma estação meteorológica automática instalada na área experimental forneceu os elementos meteorológicos: velocidade do vento (O14A, MET ONE INSTRUMENTS, Grants Pass, OR), temperatura e umidade relativa do ar (HMP35C, VAISALA, Woburn, MA) coletados a 2 m de altura. Foram armazenados valores médios de $30 \mathrm{~min}$, a partir de medidas obtidas com frequiência de $1 \mathrm{~Hz}$, e também os valores extremos de temperatura e umidade relativa do ar, por um datalogger (CR10X, CAMPBELL SCIENTIFIC Inc., Logan, UT).

$\mathrm{Na}$ avaliação da influência dos métodos de cálculo de $\mathrm{D}_{\text {ar_méd, }}$ na estimativa de $\mathrm{ET}_{\mathrm{o}}$ pela eq.(1), os seguintes coeficientes estatísticos foram utilizados: média aritmética, variância, coeficiente de determinação $\left(\mathrm{R}^{2}\right)$, interceptação (a) e coeficiente angular (b) da regressão linear entre $\mathrm{ET}_{\mathrm{o}}$, medida pelo lisímetro (X) e estimada por Penman-Monteith (Y). Utilizaram-se, também, o erro-padrão de estimativa (EPE) e o coeficiente de sensibilidade relativa (Sr). Efetuaram-se os testes t e F para médias e variâncias de $\mathrm{ET}_{\mathrm{o}}$, respectivamente, em função dos vários métodos e para os valores medidos pelo lisímetro.

$\mathrm{Na}$ análise de sensibilidade da eq.(1), utilizou-se a metodologia descrita por McCUEN (1973), MELLO (1998) e HUPET \& VANCLOOSTER (2001). A eq.(1) necessita de diversos elementos de entrada que podem introduzir erros. Uma forma geral de se expressar $\mathrm{ET}_{\mathrm{o}}$ é a seguinte:

$$
\mathrm{ET}_{\mathrm{o}}=f\left(v_{1}+v_{2}+\ldots+v_{n}\right)
$$

em que, $v_{1}, v_{2}, \ldots v_{n}$, são os $n$ elementos de entrada.

Se $\Delta v_{i}$ é a perturbação do elemento $i$, então a perturbação em $\mathrm{ET}_{\mathrm{o}}$ como resultado da perturbação de todos os elementos, pode ser quantificada por:

$$
\mathrm{ET}_{\mathrm{o}}+\Delta \mathrm{ET}_{\mathrm{o}}=f\left(v_{1}+\Delta v_{1}+v_{2}+\Delta v_{2}+\ldots+v_{n}+\Delta v_{n}\right)
$$


TABELA 1. Descrição dos grupos e métodos de estimativa da média diária do déficit de pressão de saturação do ar ( $\left.\mathrm{D}_{\text {ar_méd }}\right)$.

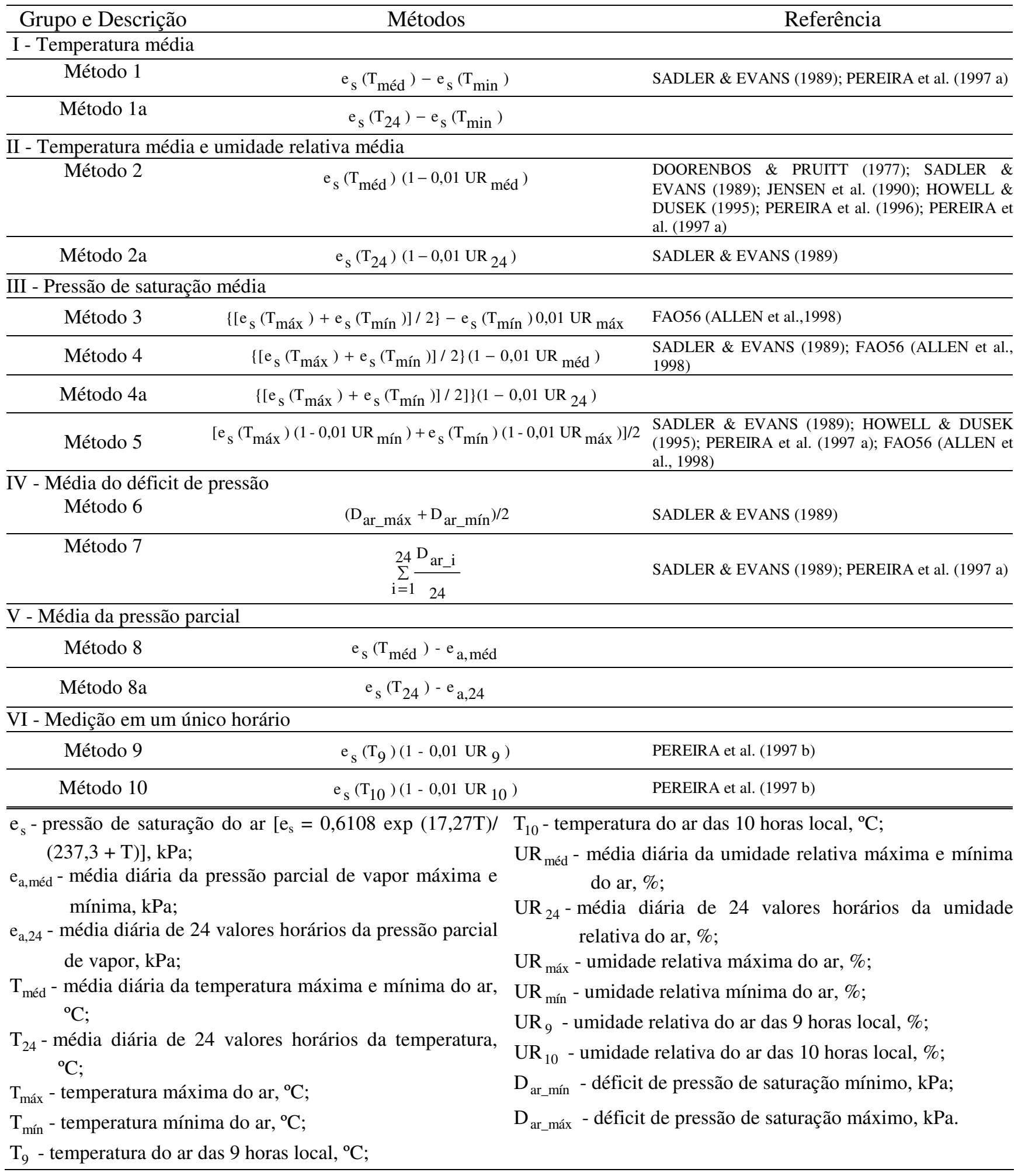


Expandindo-se a eq.(3) em uma série de Taylor e ignorando os termos de segunda ordem e as potências maiores, pode-se escrever:

$$
\Delta \mathrm{ET}_{\mathrm{o}}=\frac{\partial \mathrm{ET}_{\mathrm{o}}}{\partial v_{1}} \Delta v_{1}+\frac{\partial \mathrm{ET}_{\mathrm{o}}}{\partial v_{2}} \Delta v_{2}+\ldots+\frac{\partial \mathrm{ET}_{\mathrm{o}}}{\partial v_{n}} \Delta v_{n}
$$

em que as derivadas parciais definem a sensibilidade da estimativa para cada elemento $i\left(v_{i}\right)$. Esses coeficientes de sensibilidade são sensíveis às magnitudes relativas de $\mathrm{ET}_{\mathrm{o}} \mathrm{e} v_{i}$. A eq.(4) assume que a perturbação de cada $v_{i}$ é independente daquela dos outros elementos. Embora haja correlação de $v_{i} \mathrm{e}$ $\mathrm{ET}_{\mathrm{o}}$, assume-se que não há correlação entre os elementos (HUPET \& VANCLOOSTER, 2001). No caso do déficit de pressão de saturação, objetivo deste estudo, um coeficiente de sensibilidade relativa adimensional (Sr), de acordo com McCUEN (1973), pode ser expresso da seguinte forma:

$$
\mathrm{Sr}=\left(\frac{\partial \mathrm{ET}_{\mathrm{o}}}{\partial \mathrm{D}_{\text {ar_méd }}}\right) \frac{\mathrm{D}_{\text {ar_méd }}}{\mathrm{ET}_{\mathrm{o}}}
$$

Diferenciando-se a eq.(1) com respeito a $\mathrm{D}_{\text {ar_méd }}$ e substituindo-se na eq.(5), obtém-se:

$$
\mathrm{Sr}=\left\{900 \frac{\gamma \mathrm{u}_{2}}{\left[\mathrm{~s}+\gamma\left(1+0,34 \mathrm{u}_{2}\right)\right]\left(\mathrm{T}_{\text {méd }}+273\right)}\right\} \frac{\mathrm{D}_{\text {ar_méd }}}{\mathrm{ET}_{\mathrm{o}}}
$$

Em cada dia analisado, há um valor de Sr para cada método de estimativa de $\mathrm{D}_{\text {ar_méd. Quanto }}$ menor o $\mathrm{Sr}$, menor será o erro repassado à $\mathrm{ET}_{\mathrm{o}}$ pelo método de estimativa de $\mathrm{D}_{\text {ar_méd. }}$

\section{RESULTADOS E DISCUSSÃO}

A média da evapotranspiração de referência medida no lisímetro $\left(\mathrm{ET}_{\mathrm{o} \_l i s}\right)$ foi $3,56 \mathrm{~mm}$ dia $^{-1}$ com variância de $1,85 \mathrm{~mm} \mathrm{dia}^{-1}$ (Tabela 2), enquanto as médias estimadas de $\mathrm{ET}_{\mathrm{o}}$ por Penman-Monteith FAO56 (PM-FAO56), utilizando os diversos métodos de cálculo da média diária do déficit de pressão de saturação do vapor d'água, variaram entre 3,36 (método 9) e 4,19 mm dia ${ }^{-1}$ (método 3). Com exceção do

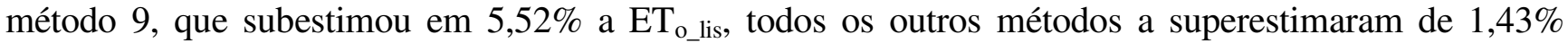
(método 2a) a 17,67\% (método 3). As médias de $\mathrm{ET}_{\mathrm{o}}$ dos métodos 3, 5 e 6 diferiram estatisticamente da $\mathrm{ET}_{\mathrm{o} \_ \text {lis }}$ pelo teste $\mathrm{t}(\mathrm{p}<0,05)$, sendo os métodos 3 e 5 recomendados pelo boletim FAO56.

As variâncias de $\mathrm{ET}_{\mathrm{o}}$ estimadas ficaram entre $1,19 \mathrm{~mm} \mathrm{dia}^{-1}$ (método 1) a 1,83 $\mathrm{mm} \mathrm{dia}^{-1}$ (método 10). As variâncias das $\mathrm{ET}_{\mathrm{o}}$ estimadas para seis métodos apresentaram diferenças significativas com relação à variância do lisímetro pelo teste $\mathrm{F}$ a p $<0,05$ (Tabela 2). Porém, seus valores não se afastaram muito do valor observado do lisímetro. Isso mostra que o manejo do lisímetro foi adequado.

Em trabalho similar, MELLO (1998) avaliou a influência dos métodos 1; 1a; 2; 2a e 5 sobre a estimativa dos valores de $\mathrm{ET}_{\mathrm{o}}$ para Córdoba, Espanha. Independentemente do método, todos subestimaram de 2,25\% a 17,64\% a $\mathrm{ET}_{\mathrm{o} \_ \text {lis. }}$ A variância de $\mathrm{ET}_{\mathrm{o} \_l \text { lis }}\left(4,26 \mathrm{~mm} \mathrm{dia}^{-1}\right)$ foi superior a dos valores estimados $\left(1,65\right.$ a 2,28 $\left.\mathrm{mm} \mathrm{dia}^{-1}\right)$. Problemas no manejo do lisímetro foi uma provável causa atentada por MELLO (1998).

O erro-padrão de estimativa (EPE) dá pesos iguais às diferenças absolutas entre valores medidos e estimados. O EPE variou entre 0,40 (método 2a) e $0,80 \mathrm{~mm} \mathrm{~d}^{-1}$ (método 3). $\mathrm{A} \mathrm{ET}_{\mathrm{o}}$ estimada em função dos métodos, utilizando a média horária da temperatura e/ou umidade relativa do ar (1a; 2a; 4a, e 8a) resultou em menores valores de EPE, em comparação aos seus híbridos com média dos valores máximo e mínimo desses elementos (métodos $1 ; 2 ; 4$, e 8). Entretanto, não foram observadas diferenças significativas entre esses métodos (teste $\mathrm{t} ; \mathrm{p}<0,05$ ). Os métodos do grupo VI obtiveram erro-padrão de estimativa entre os menores determinados, sendo de 0,44 (método 9) e 0,50 $\mathrm{mm} \mathrm{dia}^{-1}$ (método 10). Dos métodos propostos pelo FAO56, apenas o EPE do método $4\left(0,48 \mathrm{~mm} \mathrm{dia}^{-1}\right)$ se encontrou na faixa dos 
menores valores, enquanto os métodos 3 e 5 apresentaram os maiores valores observados, sendo, respectivamente, 0,80 e $0,78 \mathrm{~mm} \mathrm{dia}^{-1}$.

TABELA 2. Média aritmética $\left(\mathrm{mm} \mathrm{dia}^{-1}\right)$ e variância $\left(\mathrm{mm} \mathrm{dia}^{-1}\right)$ da evapotranspiração de referência medida pelo lisímetro e estimada pela equação de Penman-Monteith (FAO56), utilizando os

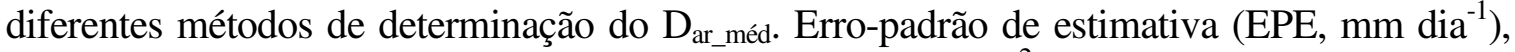
sensibilidade relativa $(\mathrm{Sr})$, coeficiente de determinação $\left(\mathrm{R}^{2}\right)$, coeficiente angular (b) e interceptação (a) da regressão linear entre os valores estimados e medidos, para os métodos avaliados.

\begin{tabular}{lccccccc}
\hline Método $^{+}$ & Média & Variância & EPE & Sr & $\begin{array}{c}\mathrm{a} \\
\left(\mathrm{mm} \mathrm{dia}^{-1}\right)\end{array}$ & $\mathrm{b}$ & $\mathrm{R}^{2}$ \\
\hline Lisímetro & 3,56 & 1,85 & & & & & \\
Método 1 & 3,85 & $1,19^{* *}$ & 0,53 & 0,31 & $1,1198 \pm 0,0741$ & $0,7682 \pm 0,0195$ & 0,9175 \\
Método 1a & 3,78 & $1,23^{* *}$ & 0,50 & 0,29 & $1,0135 \pm 0,0786$ & $0,7778 \pm 0,0206$ & 0,9103 \\
Método 2 & 3,71 & $1,32^{* *}$ & 0,43 & 0,28 & $0,8224 \pm 0,0742$ & $0,8119 \pm 0,0195$ & 0,9253 \\
Método 2a & 3,61 & 1,41 & 0,40 & 0,25 & $0,6321 \pm 0,0791$ & $0,8367 \pm 0,0208$ & 0,9206 \\
Método 3 & $4,19^{*}$ & $1,31^{* *}$ & 0,80 & 0,36 & $1,3764 \pm 0,0941$ & $0,7902 \pm 0,0247$ & 0,8795 \\
Método 4 & 3,79 & $1,33^{* *}$ & 0,48 & 0,29 & $0,9012 \pm 0,0782$ & $0,8122 \pm 0,0205$ & 0,9178 \\
Método 4a & 3,70 & 1,43 & 0,45 & 0,27 & $0,7190 \pm 0,0856$ & $0,8380 \pm 0,0225$ & 0,9085 \\
Método 5 & $4,17 *$ & 1,43 & 0,78 & 0,36 & $1,2269 \pm 0,0983$ & $0,8269 \pm 0,0258$ & 0,8800 \\
Método 6 & $4,16^{*}$ & 1,43 & 0,77 & 0,35 & $1,2296 \pm 0,0977$ & $0,8248 \pm 0,0257$ & 0,8806 \\
Método 7 & 3,82 & 1,46 & 0,51 & 0,29 & $0,8221 \pm 0,0908$ & $0,8421 \pm 0,0238$ & 0,8990 \\
Método 8 & 3,78 & $1,40^{* *}$ & 0,48 & 0,29 & $0,8334 \pm 0,0856$ & $0,8278 \pm 0,0225$ & 0,9065 \\
Método 8a & 3,70 & 1,44 & 0,44 & 0,27 & $0,7044 \pm 0,0837$ & $0,8423 \pm 0,0220$ & 0,9130 \\
Método 9 & 3,36 & 1,72 & 0,44 & 0,18 & - & $0,9233 \pm 0,0239$ & 0,9145 \\
Método 10 & 3,78 & 1,83 & 0,50 & 0,27 & $0,4317 \pm 0,1049$ & $0,9413 \pm 0,0275$ & 0,8928 \\
\hline
\end{tabular}

* A média do valor estimado difere estatisticamente da média do lisímetro pelo teste $\mathrm{t}(\mathrm{p}<0,05)$

** A variância do valor estimado difere estatisticamente da variância dos valores do lisímetro pelo teste $\mathrm{F}(\mathrm{p}<0,05)$

- Interceptação não significativa $(\mathrm{p}<0,05)$

${ }^{+}$Conforme descrito na Tabela 1

MELLO (1998) encontrou valores de EPE superiores aos determinados no presente trabalho, variando de 0,80 (método 5) a 1,25 $\mathrm{mm} \mathrm{dia}^{-1}$ (método 2a). Aqui, o menor valor de EPE dos métodos similares aos avaliados por MELLO (1998) foi o do método $2 \mathrm{a}\left(0,40 \mathrm{~mm} \mathrm{dia}^{-1}\right)$, e o maior foi obtido pelo método $5\left(0,78 \mathrm{~mm} \mathrm{dia}^{-1}\right)$. Porém, também não foram observadas diferenças significativas entre $\mathrm{ET}_{\mathrm{o}} \mathrm{em}^{\mathrm{m}}$

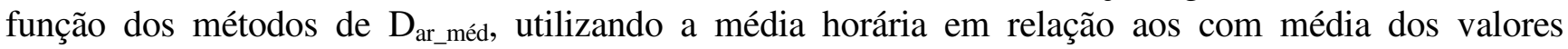
máximo e mínimo da temperatura e/ou umidade relativa do ar.

Pela análise do coeficiente de sensibilidade relativa ( $\mathrm{Sr}$ ), observou-se que a $\mathrm{ET}_{\mathrm{o}}$ estimada foi menos sensível ao método $9(0,18)$ e mais sensível aos métodos 3 e $5(0,36)$. Esses resultados significam que erros de $10 \%$ na estimativa do $\mathrm{D}_{\text {ar_méd }}$ implica incremento de 1,8\% na $\mathrm{ET}_{\mathrm{o}}$ pelo método 9 e de 3,6\% no caso dos métodos 3 e 5. Não houve $\mathrm{Sr}$ negativo, o que indicaria redução na $\mathrm{ET}_{\mathrm{o}}$ com incremento no $\mathrm{D}_{\text {ar_méd. }}$.

Esses valores são semelhantes àqueles encontrados por SAXTON (1975), para a equação de Penman $(0,20 \leq \mathrm{Sr} \leq 0,30)$, e por MELLO (1998) e BASELGA (1990), para o método de PenmanMonteith, cujos valores encontrados foram, respectivamente, 0,32 a 0,41 e 0,25 a 0,50. 
Em termos de comparação entre valores medidos e preditos por modelos, WILLMOTT (1981) sugere que sejam utilizados a interceptação (a) e o coeficiente angular (b) da regressão entre valores observados e estimados, bem como uma análise gráfica da dispersão dos valores em torno da reta 1:1. Quanto mais próximo de 1 for o valor do coeficiente angular e menor interceptação, melhor ajuste do modelo.

Os coeficientes de determinação $\left(\mathrm{R}^{2}\right)$ entre o valor medido e estimado (Tabela 2$)$ foram elevados, ficando entre 0,8800 e 0,9253, para os métodos 5 e 2, respectivamente. Os métodos dos grupos II, V e VI obtiveram coeficientes angulares próximos de 1 , variando entre 0,8119 $\pm 0,0195$ (método 2) e 0,9413 \pm 0,0275 (método 10), com baixos valores de interceptação, entre 0,4317 $\pm 0,1049$ (método 10) e 0,8334 \pm $0,0856 \mathrm{~mm} \mathrm{~d}^{-1}$ (método 8), sendo a interceptação do método 9 não-significativa $(\mathrm{p}<0,05)$. Nos métodos 3; 5 e 6, o coeficiente angular esteve na mesma faixa dos grupos supracitados, com exceção do método 3 $(0,7902 \pm 0,0247)$, que se afastou mais do ideal, porém os valores da interceptação foram os mais elevados entre todos, acima de $1,2 \mathrm{~mm} \mathrm{dia}^{-1}$. O grupo I apresentou altos valores da interceptação e coeficientes angulares afastados do ideal (Tabela 2).

Apesar de os métodos do grupo VI apresentarem boas estimativas de $\mathrm{ET}_{\mathrm{o}}$, deve-se tomar cuidado na sua utilização, principalmente por esses poderem proporcionar erros não-sistemáticos elevados; entretanto, é uma alternativa prática para locais com condições climáticas similares às da área em estudo.

O método 7 obteve coeficiente angular de 0,8421 $\pm 0,0238$ e interceptação de 0,8221 $\pm 0,0908 \mathrm{~mm} \mathrm{dia}^{-1}$.

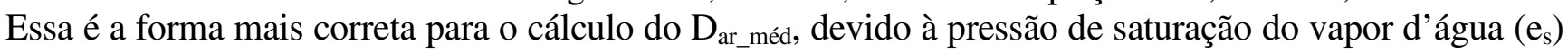
ser função exponencial da temperatura do ar. Entretanto, sua utilização para a estimativa de ET $_{0}$ superestimou os valores menores que $5,0 \mathrm{~mm} \mathrm{dia}^{-1}$ e subestimou os maiores. Esse resultado se deve às parametrizações dos outros elementos da equação de PM-FAO56. Assim, nem sempre o método que melhor estima $\mathrm{D}_{\text {ar_méd }}$ é o mais indicado para ser utilizado em PM-FAO56.

ALLEN et al. (1998) sugerem que a pressão de saturação média seja calculada pela média aritmética da pressão de saturação à temperatura mínima e máxima do ar ([es( $\left.\left.\left.\mathrm{T}_{\text {máx }}\right)+\operatorname{es}\left(\mathrm{T}_{\text {mín }}\right)\right] / 2\right)$, como nos métodos do grupo IV, com o intuito de minimizar o problema da não-linearidade da curva de pressão de saturação. Contudo, apenas os métodos que utilizaram a média da umidade relativa do ar na pressão parcial (método 4 e 4a) apresentaram valores do coeficiente angular próximos de 1 e da intercepção baixos (Tabela 2).

A $\mathrm{ET}_{\mathrm{o}}$ estimada mostrou três tendências em função do método de $\mathrm{D}_{\text {ar_méd }}$ utilizado (Figura 1). Houve a superestimativa dos valores da $\mathrm{ET}_{\mathrm{o}}$ menores que $4,5 \mathrm{~mm} \mathrm{dia}^{-1}$, em média, e subestimativa dos valores maiores, utilizando os métodos $1 ; 1 \mathrm{a} ; 2 ; 2 \mathrm{a} ; 4 ; 4 \mathrm{a} ; 7 ; 8$ e 8a. Nos métodos 3; 5; 6 e 10 , a tendência foi sempre de superestimar, enquanto no método 9 houve sempre subestimativa. 


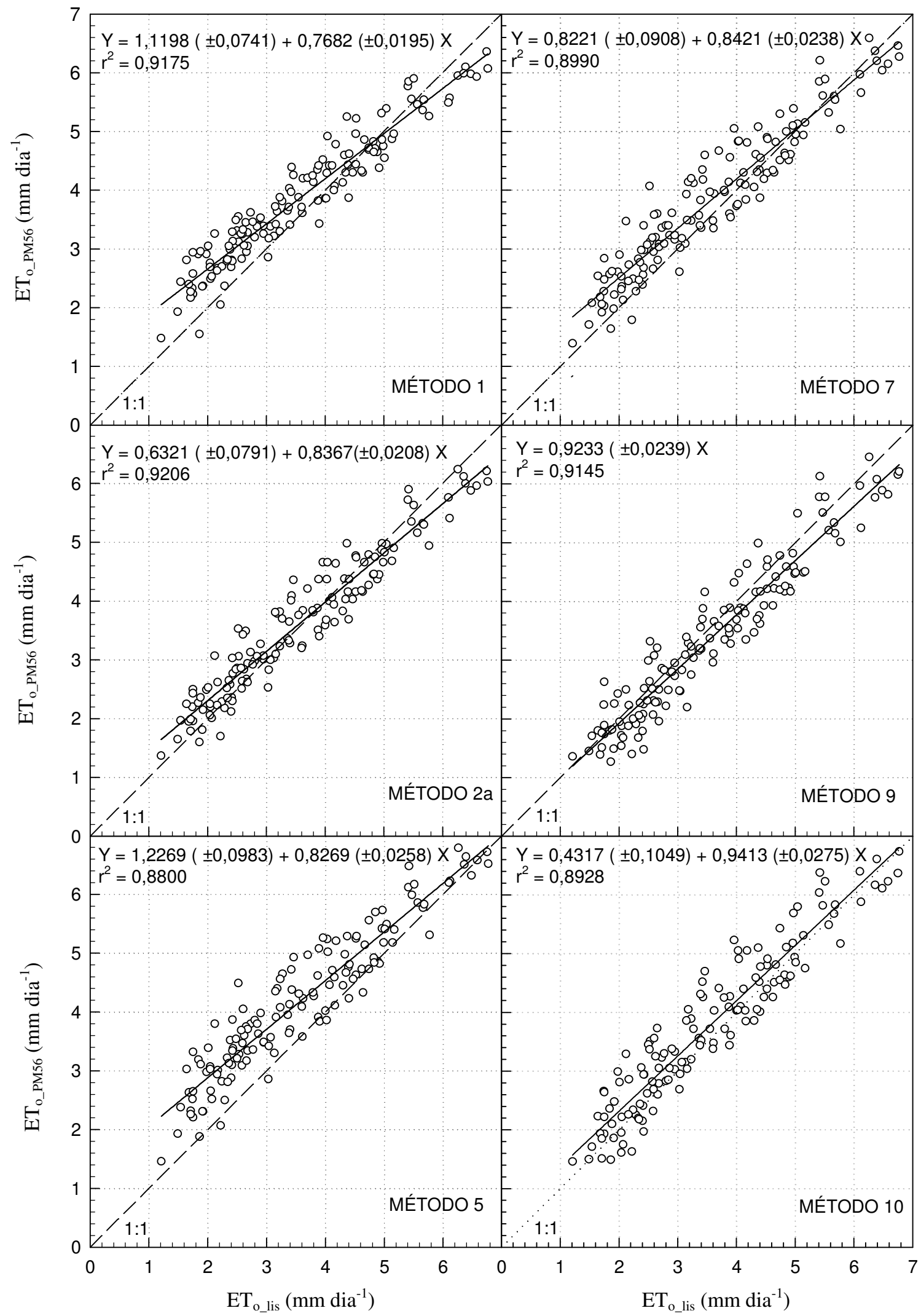

FIGURA 1. Relação entre $\mathrm{ET}_{\mathrm{o}}$ medida pelo lisímetro $\left(\mathrm{ET}_{\mathrm{o} \_l i s}\right)$ e estimada pelo modelo de PenmanMonteith FAO56 (ET __PM56), em função dos métodos 1; 2a; 5; 7; 9 e 10 (conforme descrito na Tabela 1), para a determinação da média diária do déficit de pressão de saturação do ar. 


\section{CONCLUSÕES}

Os métodos de estimativa da média diária do déficit de pressão de saturação do vapor d'água do ar afetaram de forma significativa a determinação da evapotranspiração de referência padrão FAO56, de modo que se torna necessário determinar os que melhor estimem a $\mathrm{ET}_{\mathrm{o}}$ para condições climáticas específicas.

Não necessariamente o melhor método de estimativa da média do déficit de pressão de saturação, como no caso do método utilizando valores horários, foi o mais indicado para ser utilizado na equação de PM-FAO56, devido às parametrizações dos outros elementos de entrada do modelo.

Independentemente de $\mathrm{D}_{\mathrm{ar} \_m e ́ d}$ ser determinado utilizando a média horária da temperatura e/ou umidade relativa do ar ou seus híbridos, com a média dos valores máximo e mínimo desses elementos, a estimativa de $\mathrm{ET}_{0}$ não apresentou diferenças significativas.

Dos métodos sugeridos pelo boletim FAO56, apenas o que utilizou a umidade relativa média do ar para estimar a pressão parcial de vapor se apresentou entre os que proporcionaram melhor estimativa de $\mathrm{ET}_{\mathrm{o}}$. Os métodos que utilizaram apenas um valor horário de $\mathrm{D}_{\mathrm{ar}}(9 \mathrm{e} 10 \mathrm{~h} \mathrm{local})$, como representativo para $\mathrm{D}_{\text {ar_méd }} \mathrm{e}$ os em função das médias diárias da temperatura e umidade relativa do ar foram os que apresentaram as melhores estimativas de $\mathrm{ET}_{0}$, seguidos dos métodos estimados com a pressão parcial média e temperatura do ar média para $\mathrm{e}_{\mathrm{s}}$. Os métodos que utilizaram a pressão de saturação à temperatura mínima do ar, para determinação da pressão parcial de vapor, não apresentaram boas estimativas para as condições climáticas da área em estudo.

\section{REFERÊNCIAS BIBLIOGRÁFICAS}

ALLEN, R.G.; PEREIRA, L.S.; RAES, D.; SMITH, M. Crop evapotranspiration: Guidelines for computing crop water requirements. Rome: FAO, 1998. 300 p. (Irrigation and Drainage Paper, 56)

BASELGA, J. The feasibility of using remotely sensed inputs for the estimation of roughness length and zero-plane displacement of corn. 1990. $119 \mathrm{f}$. Tese (Mestrado em Irrigação) - Utah State University, Logan,1990.

BAVEL, C.H.M. Van. Potential evaporation: the combination concept and its experimental verification. Water Resource Research, Washington, v.2, p.455-67, 1966.

DOORENBOS, J.; PRUITT, W.O. Crop water requirements. Rome: FAO, 1975. 179 p. (Irrigation and Drainage Paper, 24).

HOWELL, T.A.; DUSEK, D.A. Comparison of vapor-pressure-deficit calculation methods - Southern High Plains. Journal of Irrigation and Drainage Engineering, New York, v.121, n.2, p.191-8, 1995.

HUPET, F.; VANCLOOSTER, M. Effect of the sampling frequency of meteorological variables on the estimation of the reference evapotranspiration. Journal of Hydrology, Amsterdam, v. 243, p.192204, 2001.

JENSEN, M.E. Consumptive use of water and irrigation requirements. New York: ASCE, 1974. 215 p. (Reports of Technical Committee on Irrigation Water Requirements)

JENSEN, M.E.; BURMAN, R.D.; ALLEN, R.G. Evapotranspiration and irrigation water requirements. New York: ASCE, 1990. 332 p. (Manual, 70).

McCUEN, R. The role of sensitivity analysis in hydrologic modeling. Journal of Hydrology, Amsterdam, v.18, p.37-53, 1973. 
MELLO, J.L.P. Análise de sensibilidade dos componentes da equação de Penman-Monteith-FAO. 1998. 78 f. Tese (Doutorado em Irrigação e Drenagem) - Universidade Federal de Viçosa, Viçosa MG, 1998.

PEREIRA, A.R.; MANIERO, M.A.; VILLA NOVA, N.A.; BARBIERI, V. Penman's wind function for a tropical humid climate. Revista Brasileira de Agrometeorologia, Santa Maria, v.4, n.1, p.69-75, 1996.

PEREIRA, A.R.; VILLA NOVA, N.A.; SEDIYAMA, G.C. Evapo(transpi)ração. Piracicaba: FEALQ, 1997a. 183 p.

PEREIRA A.R.; SENTELHAS, P.S.; MARCHI, J.L. Estimativa do déficit de saturação médio diário. In: REUNIÓN ARGENTINA Y LATINOAMERICANA DE AGROMETEOROLOGIA, 7., 1997. Anais... Buenos Aires: Asociación Argentina de Agrometeorología, 1997 b. p.15-16.

PEREIRA, A.R.; SANTIAGO, A.V.; MAGGIOTTO, S.R.; FOLEGATTI, M.V. Problemas operacionais com lisímetro de pesagem durante a estação chuvosa e em dias secos com rajadas de vento. Revista Brasileira de Agrometeorologia, Santa Maria, v.10, n.1, p.51-6, 2002.

PRUITT, W.O.; DOORENBOS, J. Empirical calibration, a requisite for evapotranspiration formulae based on daily or long mean climatic data? In: INTERNATIONAL ROUND TABLE CONFERENCE ON "EVAPOTRANSPIRATION”, 1977, Budapeste. Anais... Budapeste: International Commission on Irrigation and Drainage, 1977. p.1-20.

SADLER, E.J.; EVANS, D.E. Vapor pressure deficit calculations and their effect on the equation combination equation. Agricultural and Forest Meteorology, Amsterdam, v. 49, n.1, p.55-80, 1989.

SANTIAGO, A.V.; PEREIRA, A.R.; FOLEGATTI, M.V.; MAGGIOTTO, S.R. Evapotranspiração de referência medida por lisímetro de pesagem e estimada por Penman-Monteith (FAO56), nas escalas mensal e decendial. Revista Brasileira de Agrometeorologia, Santa Maria, v.10, n.1, p.57-66, 2002.

SAXTON, K. E. Sensitivity analyses of the combination evapotranspiration equation. Agricultural Meteorology, Amsterdam, v.15, n. , p.343-53, 1975.

SILVA, F.C.; FOLEGATTI, M.V.; PEREIRA, A.R.; VILLA NOVA, N.A. Uso de dispositivos lisimétricos para medida da evapotranspiração de referência. Revista Brasileira de Agrometeorologia, Santa Maria, v.7, n.1, p.19-23, 1999 a.

SILVA, F.C.; FOLEGATTI, M.V.; MAGGIOTTO, S.R. Análise do funcionamento de um lisímetro de pesagem com célula de carga. Revista Brasileira de Agrometeorologia, Santa Maria, v.7, n.1, p.53-8, 1999 b.

WILLMOTT, C. J. On the validation of models. Physical Geography, Palm Beach, v.2, n.2, p.184-94, 1981. 